\title{
An investigation using lectins of glycocomponents of mouse spermatozoa during capacitation and sperm-zona binding
}

\author{
S. H. Lee and K. K. Ahuja \\ MRC Experimental Embryology and Teratology Unit, Woodmansterne Road, Carshalton, \\ Surrey SM5 4EF, U.K.
}

\begin{abstract}
Summary. Ten different lectins conjugated to fluorescein isothiocyanate (FITC) were used to study the distribution of surface carbohydrates on mouse spermatozoa, and to monitor the possible changes of their distribution during capacitation in vitro and sperm-egg interaction. Most of the lectins gave a restricted pattern of binding to fixed or unfixed epididymal spermatozoa. Binding was highly specific because no staining occurred in the presence of appropriate monosaccharides. Binding of UEA I, DBA and Con A was unaffected by the type of fixative used, but it was influenced by mild centrifugation. While unwashed spermatozoa showed binding mainly over the acrosomal cap and equatorial or postacrosomal regions, spermatozoa washed by mild centrifugation showed a change in the staining of the equatorial segment. Binding of 5 different lectins to spermatozoa did not change during capacitation in vitro. In contrast, capacitated spermatozoa bound to the zona pellucida exhibited a UEA I binding pattern which was strikingly different from that of the capacitated but unbound spermatozoa. We conclude that glycocomponents of specific regions of mouse spermatozoa do not change dramatically during capacitation, but do alter significantly during binding to the zona pellucida.
\end{abstract}

\section{Introduction}

Mammalian fertilization involves a sequence of interactions between the male and female gametes which include sperm attachment to the zona pellucida, sperm binding to and penetration through the zona and finally sperm - egg fusion. Before these interactions however, epididymal spermatoza must undergo certain physiological changes in the female reproductive tract which are called collectively sperm capacitation (Chang, 1951; Austin, 1952). Studies using in-vitro methods of sperm capacitation have indicated that capacitation is a multifaceted membrane phenomenon involving changes in intracellular (Yanagimachi, 1981; Fraser, 1984b) as well as surface components (Ahuja, 1985) which include surface glycocomponents. For example, temporal changes in surface carbohydrates of hamster spermatozoa have been detected in the head and midpiece regions, and in motile spermatozoa these changes appear to be under the direct control of intracellular changes (Ahuja, 1984, 1985). Surface antigens of guinea-pig spermatozoa, which are apparently involved in regulating interactions with the zona pellucida, undergo significant migration not only during capacitation but also during binding to the zona pellucida (Primakoff $e t$ al., 1985).

An investigation of similar mechanisms in the mouse is required because most biochemical studies with regard to sperm-zona recognition mechanisms have been carried out in this species, resulting in a number of working hypotheses which involve surface glycoproteins (Ahuja, 1982; Shur \& Hall, 1982; Lambert, 1984; Wassarman et al., 1985). We have therefore studied the distribution of glycosylated components on the surface of mouse spermatozoa during capacitation and sperm-zona interactions. 


\section{Materials and Methods}

Materials. Lectins conjugated to fluorescein isothiocyanate (FITC) were obtained from E-Y Lab (San Mates, CA, U.S.A.), Vector Laboratories (Burlingame, CA, U.S.A.) and Sigma (St Louis, MO, U.S.A.). Con A- and WGA-coated agarose beads were obtained from E-Y Lab. All other chemicals including inhibitory sugars were of analytical grade and were obtained from Sigma.

Sperm collestion and culture. Spermatozoa were collected from the cauda epididymidis of F1 (C57BL/6Lac $\times$ $\mathrm{CBA} / \mathrm{CaLac}$ ) hybrid males of proven fertility by incising the epididymis and releasing the sperm mass into $1 \mathrm{ml} \mathrm{T} 6$ medium (Quinn et al., 1982) containing $15 \mathrm{mg} \mathrm{BSA} / \mathrm{ml}$ (fraction V, Sigma) under paraffin oil in a plastic tissue culture dish $(35 \times 10 \mathrm{~mm}$, Falcon Plastics No. 3001F, Oxford, U.K.). The spermatozoa were allowed to disperse for 10-15 min before removing cell debris and large aggregations of immotile spermatozoa from the culture drop with a Pasteur pipette under the dissecting microscope. The sperm concentration was determined in these 'cleaned' preparations with a haemocytometer and aliquants of the sperm suspension were diluted under oil to give a final concentration of $2-5 \times 10^{6}$ spermatozoa/ml. The culture dishes containing motile spermatozoa were then incubated for $2 \mathrm{~h}$ at $37^{\circ} \mathrm{C}$ in $5 \% \mathrm{CO}_{2}$ in air to allow sperm capacitation to take place.

Egg collection. F1 (C57BL/6Lac $\times \mathrm{CBA} / \mathrm{CaLac})$ hybrid females were superovulated at $6-8$ weeks of age by intraperitoneal injections of 7.5 i.u. PMSG (Folligon: Intervet, Cambridge, U.K.) followed $48-54 \mathrm{~h}$ later with 5 i.u. hCG (Chorulon: Intervet). About $14-16 \mathrm{~h}$ later, cumulus masses containing eggs were released from the tubal ampullae into M2 medium (Quinn et al., 1982) containing $4 \mathrm{mg}$ crystalline BSA/ml (Miles Scientific, Slough, U.K.). Cumulus cells were separated from oocytes by treatment with hyaluronidase ( $150 \mathrm{units} / \mathrm{ml})$ for about $10 \mathrm{~min}$. Ova were washed three times in $2 \mathrm{ml} \mathrm{M} 2$ medium and then incubated for in-vitro fertilization in Medium $\mathrm{T} 6$ at $37^{\circ} \mathrm{C}$ in $5 \%$ $\mathrm{CO}_{2}$ in air. Zona fragments were prepared by mechanically rupturing the zonae pellucidae of ovulated ova using a finely drawn Pasteur pipette and washed three times in M2 medium.

FITC-lectin labelling. Aliquants of sperm suspension were diluted to a concentration of $2-5 \times 10^{6} / \mathrm{ml} 15 \mathrm{~min}$ after sperm preparation (fresh spermatozoa) and also after incubation for $2 \mathrm{~h}$ (capacitated spermatozoa). FITC-lectin labelling was initially carried out on unfixed spermatozoa and spermatozoa fixed in $2 \%$ paraformaldehyde. Unfixed and paraformaldehyde-fixed spermatozoa were washed by centrifugation at $700 \mathrm{~g}$ for $7 \mathrm{~min}$. The sperm pellet was suspended in PBS containing $15 \mathrm{mg} \mathrm{BSA} / \mathrm{ml}$ and washed. The pellet was resuspended in PBS + BSA $(4 \mathrm{mg} / \mathrm{ml})$ to the original concentration of the sperm suspension. Then $50 \mu \mathrm{l}$ of diluted FITC-lectins were added to the same volume of resuspended spermatozoa so that the final concentration of lectins was $25-250 \mu \mathrm{g} / \mathrm{ml}$ (Table 1). Spermatozoa were mixed well with lectins by gentle agitation and incubated at room temperature for $30 \mathrm{~min}$. Afterwards unbound FITC-lectins were removed by washing and centrifugation as described above. Spermatozoa were resuspended in a small volume of PBS, mounted on a slide with a drop of oxidized $p$-phenylenediamine $(1 \mathrm{mg} / \mathrm{ml})$ in $90 \%$ glycerol buffered with carbonate (pH 8.0) as an antifading mounting medium (Oriol \& Mancilla-Jimenex, 1983). This medium gave excellent background colour enabling the different regions of the sperm head to be visualized clearly when weak tungsten light was mixed with u.v. light. Spermatozoa were observed using epifluorescence on a Leitz Ortholux II microscope with the appropriate filter for FITC fluorescence. Photographs were taken with Ilford HP5 film.

Table 1. Lectins conjugated to fluorescein isothiocyanate (FITC) for labelling mouse spermatozoa

\begin{tabular}{|c|c|c|c|}
\hline Source & Abbreviation & $\begin{array}{l}\text { Conc. } \\
(\mu \mathrm{g} / \mathrm{ml})\end{array}$ & $\begin{array}{c}\text { Sugar } \\
\text { specificity* }\end{array}$ \\
\hline Bandeirea simplicifolia & BSL II & 25 & $\alpha / \beta$-D-GlcNAc ${ }^{\dagger}$ \\
\hline Concanavalia ensiformis & Con $\mathrm{A}$ & 25 & $\begin{array}{l}\alpha-D-M a n \dagger \\
\alpha \text {-D-Glc-D-GlcNAc }\end{array}$ \\
\hline Dolichos biflorus & DBA & 25 & $\alpha$-D-GalNAc $\dagger$ \\
\hline Lotus tetragonolobus & FBP & 250 & $\alpha$-L-Fuc $\dagger^{\dagger}$ \\
\hline Limulus polyphemus & LPA & 250 & NANA $\dagger$ \\
\hline Maclura pomifera & MPA & 25 & $\begin{array}{l}\alpha-D-G a l \dagger \\
\text { D-GalNAc }\end{array}$ \\
\hline Arachis hypogaea & PNA & 25 & $\begin{array}{l}\beta \text {-D-Gal-GalNAc } \\
\text { D-Gal } \dagger\end{array}$ \\
\hline Ricinus communis & RCA I & 25 & $\begin{array}{l}\beta \text {-D-Gal } \dagger \\
\alpha \text {-D-Gal }\end{array}$ \\
\hline Ulex europaeus & UEA I & 250 & $\alpha$-L-Fuc $\dagger$ \\
\hline Triticum vulgaris & WGA & 25 & $\begin{array}{l}\alpha / \beta-D-G l c N A c \dagger \\
\text { NANA }\end{array}$ \\
\hline
\end{tabular}

*Abbreviations of saccharides are Fuc, fucose; Gal, galactose; Glc, glucose; GlcNAc, $\mathrm{N}$-acetylglucosamine; GalNAc, N-acetylgalactosamine; Man, mannose; NANA, $\mathrm{N}$-acetylneuraminic acid.

†Inhibitory sugars for control experiment were used at final concentrations of $0.4 \mathrm{M}$. 
For comparison another protocol was used as follows. A drop $(20 \mu \mathrm{l})$ of the diluted sperm suspension was smeared on a slide, air-dried, and fixed in paraformaldehyde for $15 \mathrm{~min}$ or absolute acetone for $2 \mathrm{~min}$. The fixed preparations were gently washed with PBS + BSA $(4 \mathrm{mg} / \mathrm{ml})$ before covering with $100 \mu$ diluted FITC-lectins at the concentrations indicated above in a moist chamber at room temperature for $30 \mathrm{~min}$. In the control groups sperm suspensions or smear preparations were incubated with FITC-lectins in the presence of specific inhibitory sugars at $0.4 \mathrm{M}$ (Table !).

In-vitro sperm-zona interaction and fertilization. Cumulus-free ova or zona fragments $(10-15)$ were added to $10 \mu 1$ drops of capacitated sperm suspension under paraffin oil in a Falcon culture dish. After 15 and 45 min samples of eggs and zona fragments were removed and washed in PBS containing BSA, and then stained with FITC-UEA I without fixation as described for the sperm suspension. Samples of eggs were assayed for fertilization after 90 min by fixing and staining according to the methods used by Fraser (1983). To monitor the sperm surface during interaction with the zona pellucida, FITC-UEA I was used because it does not bind to the mouse zona pellucida (S. H. Lee \& K. K. Ahuja, unpublished). Sperm-egg or sperm-zona fragment complexes were therefore labelled with FITC-UEA I at $5 \mathrm{C}$ for $1 \mathrm{~h}$, after which they were washed in several changes of PBS and finally observed under the fluorescence microscope as described above.

Sperm-bead binding. Con A- and WGA-coated agarose beads ( 6 and $7 \mathrm{mg}$ lectins/ml beads, respectively) or Sepharose 5B Gel (Pharmacia Fine Chemicals, Uppsala, Sweden) were thoroughly washed in PBS before incubating with spermatozoa undergoing capacitation at $37^{\circ} \mathrm{C}$ in $5 \% \mathrm{CO}_{2}$ in air. About 15 selected beads were added to $10 \mu \mathrm{l}$ drops of sperm suspension at appropriate times during capacitation. After $10 \mathrm{~min}$ the sperm-bead complexes were taken out and washed gently in several drops of PBS. They were briefly fixed in $5 \%$ formalin in PBS before being photographed under dark-field illumination on an inverted phase-contrast Leitz microscope.

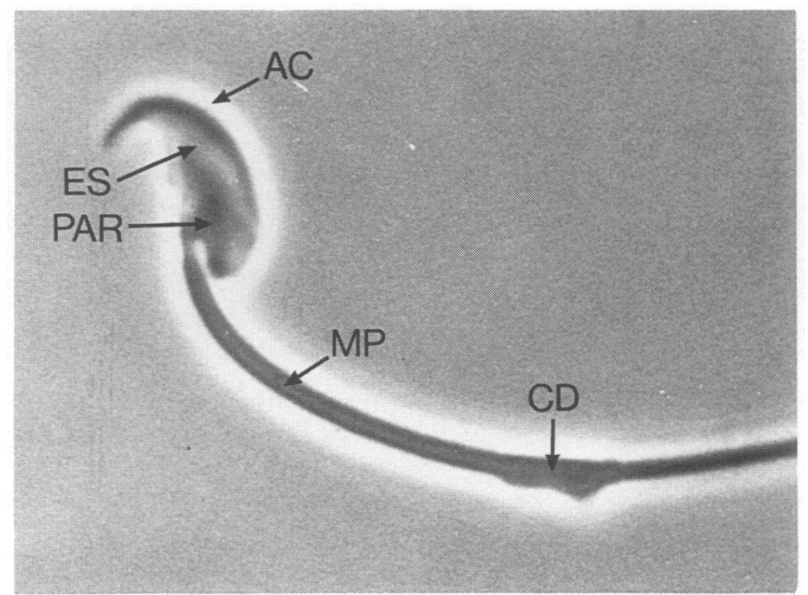

Fig. 1. A mouse spermatozoon showing different regions of head and flagellum. AC, acrosomal cap; ES, equatorial segment; PAR, postacrosomal region; MP, midpiece; CD, cytoplasmic droplet. $\times 1250$.

\section{Results}

Clear regionalized binding to the surface of epididymal mouse spermatozoa was observed with all the lectins used in this study except FITC-FBP which bound to spermatozoa rather uniformly (Figs $1 \& 2$ ). Although the fixed and unfixed spermatozoa showed binding in the same domains, the binding was more consistent in the former. Therefore, the paraformaldehyde-fixed washed spermatozoa were studied in detail for carbohydrate distribution.

\section{Labelling patterns of different FITC-lectins}

Ten different FITC-lectins gave four typical (80-95\% scored spermatozoa) binding patterns with various binding intensities. Firstly, BSL II, DBA, LPA, UEA I and Con A binding sites were largely restricted to the head region. With these lectins intensive fluorescence was observed on the 


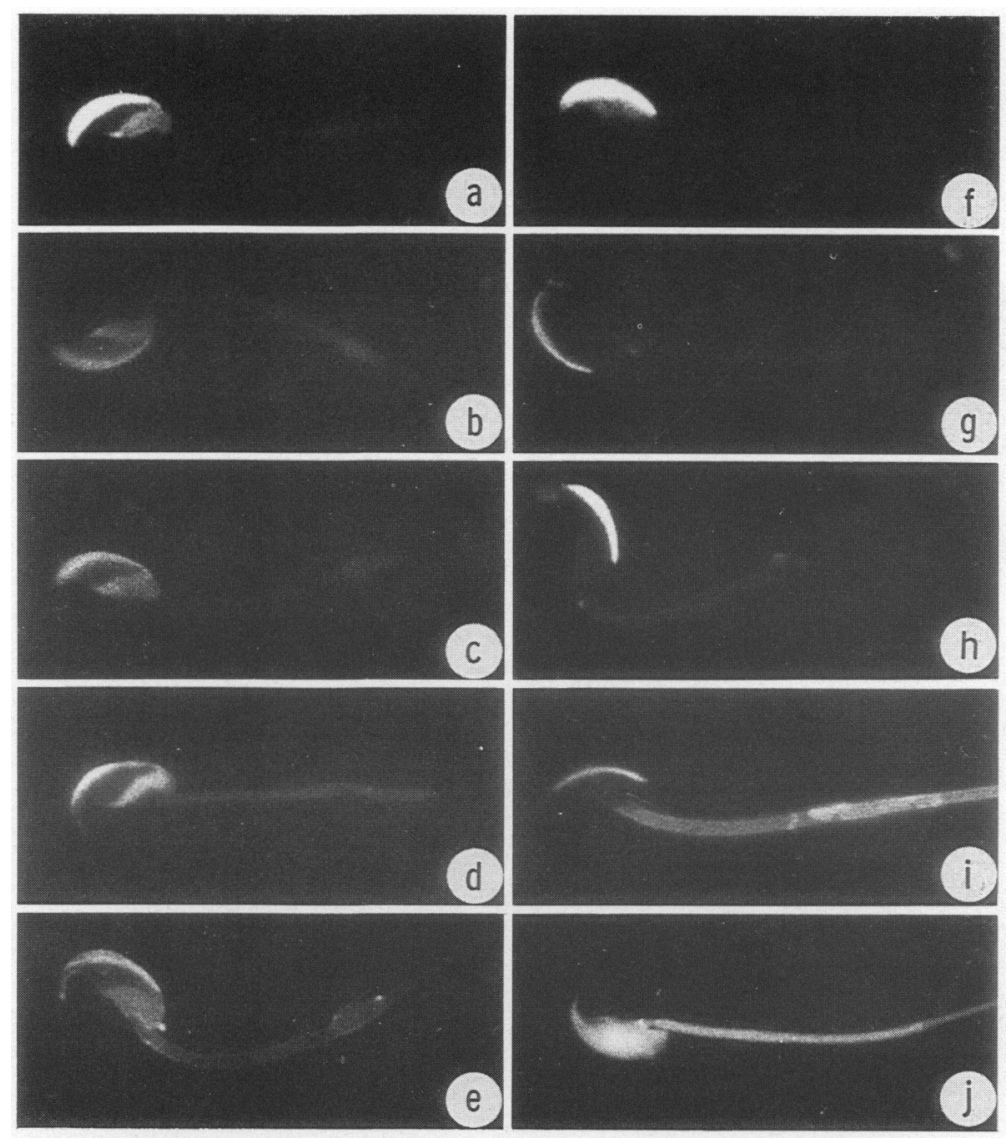

Fig. 2. Lectin binding sites on the surface of paraformaldehyde-fixed mouse spermatozoa (see 'Materials and Methods'). BSL II, DBA, LPA, UEA I and Con A (a-e) bound specifically to the acrosomal cap and postacrosomal region of the spermatozoa, the flagellar region remaining completely unstained except for weak staining on the midpiece. PNA, RCA I and WGA (f-h) showed intense binding to the acrosomal cap region only. MPA binding sites (i) were found on the acrosomal cap and the flagellar region and FBP (j) gave uniform staining of the sperm surface. $\times 625$.

acrosomal cap and postacrosomal region (Figs 2a-e). Midpieces showed weak or no staining with these lectins. Secondly, PNA, RCA I and WGA binding sites were confined to the acrosomal cap region (Figs $2 \mathrm{f}-\mathrm{h}$ ). Thirdly, MPA staining was most intense on the acrosomal cap, with the flagellar region being moderately positive (Fig. 2i). Lastly, FBP binding sites were uniformly distributed over the entire surface (Fig. 2j). Although FBP and UEA I are both fucose-specific lectins, they showed different preferences for binding sites on the sperm surface. No staining was observed in the control specimens treated with FITC-lectins in the presence of $0.4 \mathrm{M}$ inhibitory monosaccharides, indicating specific binding of lectins.

\section{Effect of labelling procedure}

The choice of a labelling procedure can sometimes have a profound influence on the pattern of cell surface staining (Peterson \& Russell, 1985). Two different labelling procedures and different fixatives were compared to see whether they can affect lectin binding sites on spermatozoa. 
Con A and UEA I were chosen for these studies because Con A has been used extensively (Millette, 1977; Schwartz \& Koehler, 1979; Koehler, 1981) and UEA I has a single specificity for fucose. In specimens smeared onto slides and then air-dried and fixed in acetone or paraformaldehyde, Con A stained the acrosomal cap strongly, the equatorial segment moderately and the midpiece weakly (Figs 3a \& 3b). Therefore, there were no differences in staining patterns between

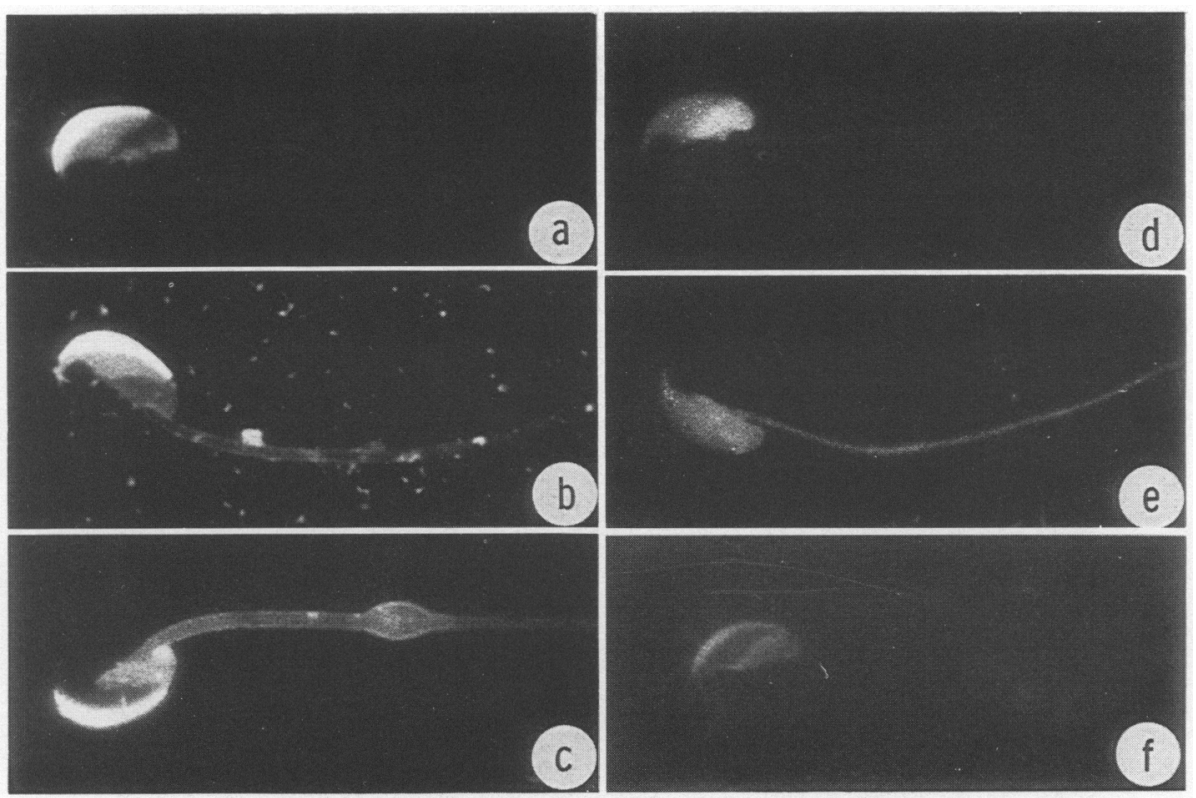

Fig. 3. Effects of fixatives and labelling procedures on the binding of Con A $(a-c)$ and UEA I $(d-f)$ to mouse epididymal spermatozoa. Air-dried smears fixed with acetone (a and d) or paraformaldehyde ( $b$ and $e$ ) stained differently from spermatozoa fixed, washed and labelled in suspension $(c$ and $f$ ). No difference was found between the two fixatives. $\times 625$.

Table 2. Binding of FITC-lectins to mouse spermatozoa undergoing capacitation in vitro*

\begin{tabular}{|c|c|c|c|c|c|}
\hline \multirow[b]{2}{*}{ Lectins } & \multicolumn{2}{|c|}{ Labelling } & \multicolumn{3}{|c|}{$\begin{array}{l}\% \text { spermatozoa } \\
\text { showing typical } \\
\text { pattern at: }\end{array}$} \\
\hline & Region† & Intensity $\ddagger$ & $15 \mathrm{~min}$ & $60 \mathrm{~min}$ & $120 \mathrm{~min}$ \\
\hline DBA & AC, PAR &,+++++ & 92 & 89 & 87 \\
\hline UEA I & AC, PAR &,+++++ & 94 & 96 & 90 \\
\hline Con $\mathrm{A}$ & AC, PAR &,+++++ & 84 & 81 & 82 \\
\hline MPA & $\mathrm{AC}, \mathrm{FL}$ &,+++++ & 89 & 96 & 91 \\
\hline FBP & EN & +++ & 92 & 93 & 98 \\
\hline
\end{tabular}

*Paraformaldehyde-fixed spermatozoa were labelled and scored as described in 'Materials and Methods'. The results were obtained from 4 experiments in each group. At least 100 spermatozoa were counted in each group. Similar results were obtained with smear preparations.

†AC, acrosomal cap; FL, flagellum; PAR, postacrosomal region; EN, entire surface.

¥Estimated subjectively on a 2-point scale denoting moderate $(++)$ and intense $(+++)$. 

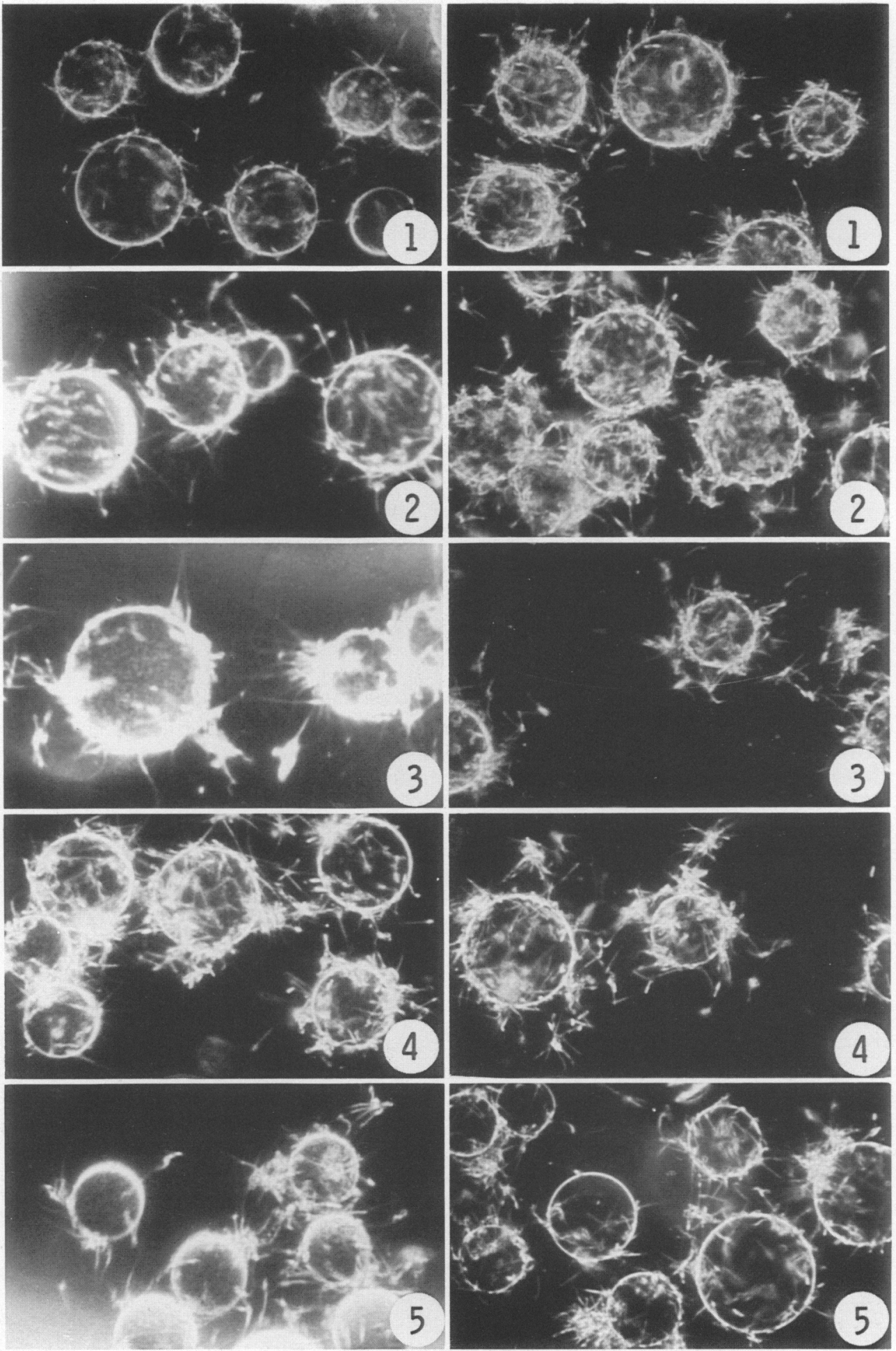


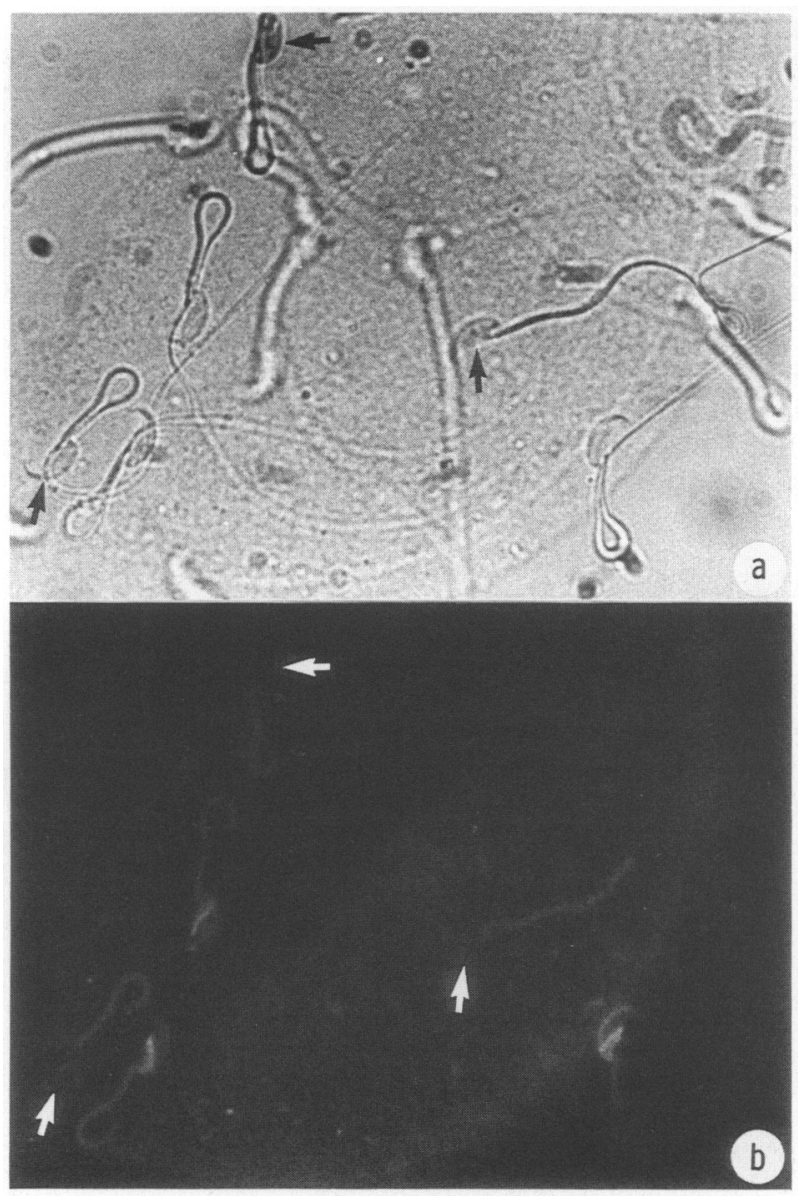

Fig. 5. FITC-UEA I binding to sperm-zona complexes. Zona fragments and cumulus free eggs were incubated with capacitated spermatozoa for $60 \mathrm{~min}$ and labelled as described in 'Materials and Methods'. Postacrosomal labelling can be seen in some spermatozoa while other spermatozoa (white arrow heads) have already lost their binding sites on the postacrosomal region. However, midpiece labelling remained on all spermatozoa. One spermatozoon reveals acrosomal cap staining (on the right). $\times 310$.

the two fixatives. In contrast, paraformaldehyde-fixed spermatozoa in suspension and washed by centrifugation showed a different pattern of lectin binding. Strong Con A binding to the acrosomal cap was still present, but the equatorial segment was completely free from any stain. Also the postacrosomal region showed moderate staining (Fig. 3c). In air-dried specimens fixed in either of the two fixatives, UEA I binding sites were similar (Figs 3d \& 3e). However, acrosomal cap staining appeared after fixation and washing by centrifugation (Fig. 3f). Similar results were obtained with another lectin, DBA, which showed staining of the acrosomal cap and postacrosomal region of spermatozoa washed by centrifugation.

Fig. 4. Sperm binding to lectin-coated beads during in-vitro capacitation. Con A- (left) and WGA-coated agarose (right) beads were incubated with epididymal spermatozoa of mouse at $30,60,90,120$ and 180 min intervals as described in 'Materials and Methods'. Numbers (1-5) indicate time intervals. No significant differences in sperm binding to beads were found. $\times 30$. 


\section{Lectin binding sites during in-vitro capacitation}

Epididymal spermatozoa incubated for 15,60 and $120 \mathrm{~min}$ were fixed in paraformaldehyde, and washed in suspension before labelling with FITC-lectins. As judged by the proportion of spermatozoa showing the characteristic binding patterns (Fig. 2), no significant differences were found between the staining properties of fresh $(15 \mathrm{~min})$ and capacitated $(120 \mathrm{~min})$ spermatozoa. The proportion of typical binding patterns remained unchanged during capacitation in vitro (Table 2). Similar results were obtained with air-dried, acetone-fixed preparations (data not shown).

Quantitative changes in lectin-binding sites during capacitation in vitro were also assessed by using an artificial non-biological surface, coated with various lectins (lectin-coated agarose beads). No differences were observed in sperm binding to Con A- or WGA-coated beads at different times during capacitation in vitro in the mouse (Fig. 4).

\section{Sperm surface changes during sperm-zona interaction}

When capacitated spermatozoa were incubated with eggs and penetration rates were scored $90 \mathrm{~min}$ later, $80-90 \%$ of eggs were penetrated. Spermatozoa bound to intact eggs or zona fragments were labelled with FITC-UEA I. This lectin was used because it shows little or no staining on the mouse zona pellucida (S. H. Lee \& K. K. Ahuja, unpublished). At 15 min after mixing with the eggs or zona fragments the spermatozoa bound to the zona showed clear staining in the postacrosomal region and occasionally weak staining in the midpiece region. This pattern is similar to that of unwashed air-dried sperm preparations labelled with FITC-UEA I or -DBA. However, between 45 and $60 \mathrm{~min}$ a small proportion of spermatozoa showed a complete absence of lectin binding sites from the postacrosomal region (Fig. 5). Due to the small size of the mouse acrosome it was not always possible to confirm the state of the acrosome in zona-bound spermatozoa, but many of them showed intact acrosomes. The moderate binding on the midpieces did not change during these interactions.

\section{Discussion}

The present results confirm a highly localized distribution of binding sites for 10 different lectins involving mainly the sperm head region. The acrosomal and postacrosomal regions appear to be rich in glycocomponents containing sugars specific for BSL II, DBA, LPA, UEA I and Con A (Figs $2 a-e)$, but the flagellar regions showed a relatively weak or moderate staining pattern except for two lectins, MPA and FBP (Figs $2 \mathrm{i} \& 2 \mathrm{j}$ ). Some of the lectins tested (PNA, RCA I and WGA) showed exclusive binding sites on the acrosomal cap (Figs $2 \mathrm{f}-\mathrm{h}$ ), similar to the binding patterns documented for spermatozoa of other mammalian species (hamster: K. K. Ahuja, unpublished; guinea-pig: Schwartz \& Koehler, 1979; human: Talbot \& Chacon, 1981). The presence of binding sites for 10 different lectins in the acrosomal region appears important, because a galactosyltransferase receptor for the zona (Shur \& Hall, 1982) as well as the binding sites for a sperm receptor protein ZP3 (Wassarman et al., 1985) are found in this region. At present, the likelihood that these different components are a part of or at least adjacent to the receptor molecule on the acrosomal region cannot be ruled out.

Two different methods of labelling the sperm surface glycoproteins were compared. One was to label the cell suspension, which required washing by centrifugation to remove unbound conjugated marker, and harvest the labelled spermatozoa. This method has been used previously in many ultrastructural and immunological investigations for other species (Koehler, 1981; TopferPetersen \& Schill, 1983). The other procedure involved labelling the air-dried smear which needs -only gentle washing by the direct application of PBS to the smeared slide. 
The staining patterns shown in Fig. 2 were obtained from cells labelled in suspension. In the case of two particular lectins, Con A and UEA I, we found that the distribution of lectin binding sites on air-dried smears was different from that of the centrifuged spermatozoa (Fig. 3). The difference may have been caused by the removal of surface-linked components from centrifuged spermatozoa as has been shown for the mouse by Fraser (1984a). A biochemical study on the plasma membrane of boar spermatozoa indicated that simple washing can remove major adsorbed proteins from the epididymal spermatozoa (Russell et al., 1983). Our experiments provide clear evidence that the components removed from the surface are probably glycoconjugates present in the acrosomal cap and postacrosomal regions of spermatozoa. Although the precise nature of these substances and the mechanism of their removal are unclear at present, it is reasonable to propose that future studies on the role of surface components in fertilization should be carried out on intact spermatozoa without centrifugation. This can probably be achieved by briefly exposing capacitating spermatozoa and then gently removing the sperm-egg complexes for washing by transfer through drops of culture media before labelling with lectins or other probes.

Surface alterations during sperm capacitation are generally believed to be an important prerequisite to successful fertilization (Koehler, 1981; Ahuja, 1984; Primakoff et al., 1985). It was therefore of interest to see whether any quantitative or qualitative changes in the distribution of binding sites for lectins occurred during sperm capacitation. With 10 different FITC-lectins, we found no significant alterations in the distribution of lectin binding sites on mouse spermatozoa capacitated in vitro (Table 2). Concentrations of spermatozoa showing typical lectin binding patterns were relatively constant at different stages of capacitation under culture conditions which result in fertilization rates of $80-90 \%$. The sperm-bead binding studies which enable the continuous monitoring of surface changes in a large proportion (Ahuja, 1984) were also undertaken. The results further confirmed that no significant alteration occurs in the lectin binding sites on mouse spermatozoa during capacitation in vitro (Fig. 4). These observations contrast with studies of spermatozoa of other species (Schwartz \& Koehler, 1979; Ahuja, 1984) and may reflect the limitation of the technique rather than the uniqueness of the mouse spermatozoa. The release of molecules containing terminal disaccharides exposing surface-linked glycosyltransferases in capacitated mouse spermatozoa has been demonstrated (Shur \& Hall, 1982), but this may be an artefact readily induced by mild centrifugation or represent a highly specific and subtle change during capacitation that presumably cannot be monitored by lectin binding studies.

Although our studies failed to detect significant changes in the distribution of lectin binding sites in capacitated spermatozoa, significant changes were observed with respect to the binding of FITC-UEA I to spermatozoa when bound to the zona (Fig. 5). Probably not all the changes in sperm components are a pre-requisite to successful binding and penetration but some alterations may occur during or after the binding between the two gametes. For example, if particular surface saccharides are involved in mediating sperm-egg recognition, they would be expected to be exposed at a precise time during fertilization under the combined control of intra- and extra-cellular factors (Fraser, 1984b; Hyne \& Edward, 1985; Shapiro et al., 1985).

The appearance and disappearance of UEA I binding sites from the acrosomal cap and postacrosomal regions of interacting spermatozoa, respectively (Fig. 5), may represent part of this phenomenon. In the mouse, the ionic permeability of the sperm membrane appears to change during binding to the zona pellucida (Lee \& Storey, 1985). Similarly, in the guinea-pig, certain antigens in the sperm membrane migrate laterally during binding to the zona pellucida (Primakoff et al., 1985). Whether these fucosylated components have any immunological properties and whether they are involved in mouse fertilization requires further work.

We thank Dr Lynn Fraser for many stimulating discussions; Dr David Whittingham for encouragement during the course of this study; David Gilburt for excellent technical assistance; and Ms Kim Hughes for typing the manuscript. 


\section{References}

Ahuja, K.K. (1982) Fertilization studies in the hamster: the role of cell surface carbohydrates. Expl Cell Res. 140, 353-362.

Ahuja, K.K. (1984) Lectin-coated agarose beads in the investigation of sperm capacitation in the hamster. Devl Biol. 104, 131-141.

Ahuja, K.K. (1985) Carbohydrate determinants involved in mammalian fertilization. Am. J. Anat. 174, 207-223.

Austin, C.R. (1952) The 'capacitation' of the mammalian sperm. Nature, Lond. 170, 326.

Chang, M.C. (1951) The fertilizing capacity of spermatozoa deposited into the Fallopian tubes. Nature, Lond. 168, 697-698.

Fraser, L.R. (1983) Mouse sperm capacitation assessed by kinetics and morphology of fertilization in vitro. $J$. Reprod. Fert. 69, 419-428.

Fraser, L.R. (1984a) Mouse sperm capacitation in vitro involves loss of a surface-associated inhibitory component. J. Reprod. Fert. 72, 373-384.

Fraser, L.R. (1984b) Mechanisms controlling mammalian fertilization. Oxford Rev. Reprod. Biol. 6, $174-225$.

Hyne, R.V. \& Edward, K.P. (1985) Influence of 2-deoxy-D-glucose and energy substrates on guineapig sperm capacitation and acrosome reaction. $J$. Reprod. Fert. 73, 59-69.

Koehler, J.K. (1981) Lectins as probes of the spermatozoan surface. Archs Androl. 6, 197-217.

Lambert, H. (1984) Role of sperm surface glycoproteins in gamete recognition in two mouse species. $J$. Reprod. Fert. 70, 281-284.

Lee, M.A. \& Storey, B.T. (1985) Evidence for plasma membrane impermeability to small ions in acrosomeintact mouse spermatozoa bound to mouse zonae pellucidae, using an aminoacridine fluorescent $\mathrm{pH}$ probe: time course of the zona-induced acrosome reaction monitored by both chlorotetracycline and pH probe fluorescence. Biol. Reprod. 33, 235-246.

Millette, C.F. (1977) Distribution and mobility of lectin binding sites on mammalian spermatozoa. In Immunobiology of Gametes, pp. 51-71. Eds M. Edidin \& M. H. Johnson. Cambridge University Press.

Oriol, R. \& Mancilla-Jimenez, R. (1983) Fluorescent staining of nuclei and amyloid substance. Two useful properties of $p$-phenylenediamine. $J$. Immunol. Methods 62, 185-192.
Peterson, R.N. \& Russell, L.D. (1985) The mammalian spermatozoon: a model for the study of regional specificity in plasma membrane organization and function. Tissue \& Cell 17, 769-791.

Primakof, P., Hyatt, H. \& Myles, D.G. (1985) A role for the migrating sperm surface antigen $\mathrm{PH}-20$ in guinea pig sperm binding to the egg zona pellucida. J. Cell Biol. 101, 2239-2244.

Quinn, P., Barros, C. \& Whittingham, D.G. (1982) Preservation of hamster oocytes to assay the fertilizing capacity of human spermatozoa. J. Reprod. Fert. 66, $161-168$.

Russell, L.D., Montag, B., Hunt, W. \& Peterson, R.N. (1983) Properties of boar sperm plasma membrane: proteins released by washing and different solubility in salts, detergents and sensitivity to surface radiolabelling. Gamete Res. 11, 237-252.

Schwartz, M.A. \& Koehler, J.K. (1979) Alterations in lectin binding to guinea pig spermatozoa accompanying in vitro capacitation and the acrosome reaction. Biol. Reprod. 21, 1295-1307.

Shapiro, B.M., Schackmann, R.W., Tombes, R.M. \& Kazazoglou, T. (1985) Coupled ionic and enzymatic regulation of sperm behaviour. Curr. Top. Cell Regulation 26, 97-113.

Shur, B.C. \& Hall, N.G. (1982) Sperm surface galactosyltransferase activities during in vitro capacitation. J. Cell Biol. 95, 567-573.

Talbot, P. \& Chacon, R. (1981) Observations on the acrosome reaction of human sperm in vitro. Am. J. Primatol. 1, 211-219.

Topfer-Petersen, E. \& Schill, W.-B. (1983) Characterization of lectin receptors isolated from the outer acrosomal membrane of boar spermatozoa. Int. J. Androl. 6, 375-392.

Wassarman, P.M., Bleil, J.D., Florman, H.M., Greve, J.M., Roller, R.J., Salzman, G.S. \& Samuels, F.G. (1985) The mouse egg's receptor for sperm: what is it and how does it work? Cold Spring Harbor Symp. quant. Biol. 50, 11-19.

Yanagimachi, R. (1981) Mechanisms of fertilization in mammals. In Fertilization and Embryonic Development in Vitro, pp. 81-182. Eds L. Mastroianni \& J. D. Biggers. Plenum Press, New York.

Received 16 July 1986 